\title{
Does mini-midvastus approach have an advantageous effect on rapid recovery protocols over medial parapatellar approach in total knee arthroplasty?
}

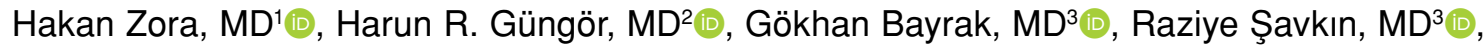 \\ Nihal Büker, $M D^{3}$ (1) \\ ${ }^{1}$ Department of Orthopedics and Traumatology, Artvin State Hospital, Artvin, Turkey \\ 'Department of Orthopedics and Traumatology, Pamukkale University Medical Faculty, Denizli, Turkey \\ ${ }^{3}$ School of Physical Therapy and Rehabilitation, Pamukkale University, Denizli, Turkey
}

Due to the demands to improve life and health conditions of patients with osteoarthritis (OA), minimally invasive surgeries have been favorable to obtain satisfactory results when performing knee arthroplasty. ${ }^{[1]}$ Rapid recovery surgical protocols are evidence-based multidisciplinary approaches targeted on multimodal patient care and primarily focused on enhancing functional recovery of patients. These protocols include patient education to cope with anxiety and stress of surgery, nutritional planning and avoidance of long hours of fasting, preemptive analgesia, avoidance of tourniquet use, rational antibiotic prophylaxis, local infiltration anesthesia, and early physical therapy modalities. The ultimate aims of assembling these surgical protocols are to

Received: May 21, 2020

Accepted: August 09, 2020

Published online: September 11, 2020

Correspondence: Harun R. Güngör, MD. Pamukkale Üniversitesi Tıp Fakültesi Ortopedi ve Travmatoloji Anabilim Dalı,

20070 Pamukkale, Denizli, Türkiye.

E-mail: hrgungor@gmail.com

Doi: $10.5606 /$ ehc. 2020.76387

Citation: Zora H, Güngör HR, Bayrak G, Şavkın R, Büker N. Does mini-midvastus approach have an advantageous effect on rapid recovery protocols over medial parapatellar approach in total knee arthroplasty? Jt Dis Relat Surg 2020;31(3):571-581.

C2020 All right reserved by the Turkish Joint Diseases Foundation

This is an open access article under the terms of the Creative Commons Attribution-NonCommercial License, which permits use, distribution and reproduction in any medium, provided the original work is properly cited and is not used for commercial purposes (http://creativecommons.org/licenses/by-nc/4.0/).

\section{ABSTRACT}

Objectives: This study aims to compare the effects of minimidvastus (MMV) versus medial parapatellar (MPP) approach on rapid recovery protocols during total knee arthroplasty (TKA).

Patients and methods: This prospective, randomized, singleblinded study was performed in 54 patients (4 males, 50 females; mean age $64.1 \pm 6.4$ years) diagnosed as primary knee osteoarthritis and planned for unilateral TKA between May 2018 and March 2019. Patients were randomly assigned as MMV (1 male, 26 females; mean age $65 \pm 6.4$ years) and MPP ( 3 males, 24 females; mean age $63.2 \pm 6.3$ years) groups. Rapid recovery TKA protocol and discharge criteria were assembled and all patients were evaluated preoperatively, and at postoperative first and third months. Length of hospital stay (LOS) was recorded for all patients. Hemoglobin and hematocrit values, radiologic assessment of alignment, knee range of motion (ROM), quadriceps muscle strength, Visual Analog Scale (VAS), 30-sec chair-stand test, stair-climb test, Western Ontario and McMaster Universities Osteoarthritis Index (WOMAC), Knee Injury and Osteoarthritis Outcome Score (KOOS), and Short Form-36 (SF-36) were used for evaluations by blinded observers.

Results: There was no significant difference in demographic variables between two groups. Operative time in MMV Group (78.1 \pm 2.7 $\mathrm{min})$ was significantly longer than the MPP Group $(65.9 \pm 2.6 \mathrm{~min})$ $(\mathrm{p}<0.0005)$. LOSs in the MMV and MPP Groups were 27.6 \pm 3.1 hours and $29.1 \pm 6.7$ hours with no significant difference. There was no statistically significant difference in postoperative measurements between groups in hemoglobin and hematocrit values, radiologic alignment of components, knee ROM, VAS, 30-sec chair-stand test, stair-climb test, WOMAC, KOOS, and SF-36 evaluations ( $p>0.05$ ). In terms of quadriceps muscle strength gain, we could not find any difference between groups in pre- and postoperative difference of changes $(\mathrm{p}>0.05)$.

Conclusion: With the use of contemporary rapid recovery protocols during TKA, MMV approach had no superiority over MPP approach when quadriceps muscle strength, LOS, pain, function, and quality of life were assessed. Longer operative time in the MMV approach compared to MPP approach may be considered as a disadvantage.

Keywords: Fast-track protocol, midvastus approach, parapatellar approach, rapid recovery protocol, total knee arthroplasty. 
decrease mortality and morbidity, length of hospital stay (LOS), and eventually hospital costs while obtaining maximum patient satisfaction. ${ }^{[2-4]}$

Surgical approaches when performing total knee arthroplasty (TKA) include standard medial parapatellar (MPP) approach and minimal invasive approaches such as mini-midvastus (MMV) and subvastus approaches. ${ }^{[2,3,5]}$ Possible advantages of not performing quadriceps tendon splitting in MV surgical approach (such as less pain, earlier functional recovery, enhanced quadriceps muscle strength, and better range of motion [ROM]) convinced surgeons to prefer minimal invasive approaches to MPP approach when performing rapid recovery protocols in TKA patients. ${ }^{[3]}$ In addition, better surgical outcomes with traditional protocols in short-term reports in favor of minimal invasive approaches also encouraged rapid recovery protocol builders to prefer minimal invasive approaches. ${ }^{[3,4,6,7]}$ However, these recommendations are not evidence based and, to our knowledge, there is no study comparing surgical outcomes between minimal invasive approaches and MPP approach in terms of pain, LOS and functional recovery in fast-track TKA patients. ${ }^{[8-12]}$ In addition, Enhanced Recovery After Surgery (ERAS ${ }^{\circledR}$ ) Society declared a consensus statement at the beginning of 2020 about perioperative care in total hip arthroplasty (THA) and TKA, and recommended that more evidence is needed to prefer one type of surgical approach over another in terms of the use of a minimally invasive technique with an ERAS $^{\circledR}$ set up. ${ }^{[13]}$ Therefore, in this study, we aimed to compare the effects of MMV versus MPP approach on rapid recovery protocols during TKA.

\section{PATIENTS AND METHODS}

This single-center, prospective, randomized, singleblinded study was conducted at the Orthopedics and Traumatology Department in Pamukkale University Medical Faculty. The study protocol was approved by the Pamukkale University Non-invasive Clinical Research Ethics Committee (Approval date and number: 06.03.2018/05). A written informed consent was obtained from each patient. The study was conducted in accordance with the principles of the Declaration of Helsinki.

Inclusion criteria were as follows: (i) age between 50 to 85 years, (ii) patients scheduled for unilateral TKA surgery due to primary OA, and (iii) patients capable of understanding verbal and written instructions. Exclusion criteria were as follows: (i) revision TKA surgery, (ii) American Society of Anesthesiologists score $>3$, (iii) previous major orthopedic surgery in either lower extremities, (iv) neurologic compromise, (v) psychiatric problems, (vi) regular hypnotic and/or anxiolytic medication usage, (vii) dementia, or (viii) patients participated in a particular physical activity program within the last three months.

Fifty-six patients were enrolled in this study between May 2018 and March 2019. Patients were randomized into two groups by a computer program to generate random numbers and assign participants to either the MMV or MPP group. Two patients were lost to follow-up and a total of 54 patients (4 males, 50 females; mean age $64.1 \pm 6.4$ years) (27 in each group) were enrolled. Mean ages of the patients in MMV and MPP groups were $65.0 \pm 6.4$ years and 63.2 \pm 6.3 years. Rapid recovery TKA protocol and discharge criteria were assembled by a multidisciplinary team comprising an orthopedic surgeon, anesthesiologist, and physiotherapists and nursing care services, and this supervised protocol was applied to all patients.

Clinical and demographic variables of the participants were recorded and patients were evaluated preoperatively, and at postoperative fourth and twelfth weeks by a blinded observer. Knee ROM was assessed with a digital goniometer (HALO Medical Devices, Perth, Australia); quadriceps muscle strength was measured (unit=newton $[\mathrm{N}]$ ) with a hand-held dynamometer (Commander Muscle Tester, JTech, Midvale, Utah, USA); Western Ontario and McMaster Universities Osteoarthritis Index (WOMAC) and Knee Injury and Osteoarthritis Outcome Score (KOOS) were used to determine patient-reported activity limitations; 30-second chair-stand test and stair-climb test were performed for performancebased activity limitations; Short Form-36 (SF-36) was used for quality of life evaluations.

Long-leg radiographs of the patients were evaluated pre- and postoperatively by using digital orthopedic templating software: Materialise OrthoView (OrthoView version 7, Materialise HQ, Technologielaan 153001 Leuven, Belgium). Hip-knee-ankle (HKA) angles, femorotibial angles, lateral proximal femoral angles (LPFA), lateral distal femoral angles (LDFA), medial proximal tibial angles (MPTA), lateral distal tibial angles (LDTA), and tibial posterior slope angles were all measured and recorded by a blinded observer.

All patients received preoperative informative classes about TKA procedure, nutritional and nursing support, and physical therapy and rehabilitation applications. Booklets concerning all these classes were also handed out to all patients. 
Excluding diabetics, all patients received oral carbohydrate $(12.5 \%$ carbohydrate liquid solution [Fantomalt, Nutricia, Hoofddorp, The Netherlands]) loading on the night before the operation (between 19:00 and 23:00) and two hours before the operation. Solid foods were allowed up to sixth preoperative hour and liquids were allowed up to second preoperative hour. Early oral feeding was started at fourth to sixth postoperative hours for all patients. Intravenous midazolam 1-2 mg and fentanyl 50-100 $\mu$ g were applied to all patients 30-45 minutes preoperatively. Except 12 patients, all patients received spinal anesthesia. Seven patients due to previous lumbar fusion and five patients due to personal preference received general anesthesia.

All operations were performed by the same surgeon using the same brand and type of prosthesis. MPP and MMV approaches were performed as described in the literature. ${ }^{[14]}$ All patients received posterior stabilized fixed bearing TKA (NexGen Legacy ${ }^{\circledR}$ Posterior Stabilized Knee-Fixed Bearing, Zimmer-Biomet Inc., Warsaw, Indiana, USA), and high viscosity polymethyl methacrylate bone cement (Oliga-G21 srl-Via S.Pertini, San Possidonio [MO], Italy). All operations were performed without using tourniquet.

Local infiltration anesthesia $(20 \mathrm{~mL}$ bupivacaine hydrochloride, $1 \mathrm{~g}$ fentanyl, $1 \mathrm{~g}$ cefazolin sodium, 0.3 $\mathrm{mL}$ epinephrine, and diluted volume of physiologic serum $[0.9 \%$ sodium chloride $(\mathrm{NaCl})]$ to $50 \mathrm{~mL}$ ) was injected to posterior capsule just before the application of permanent implants, and to anterior capsule, prepatellar fat pad and periligamentous nociceptive receptors following consolidation of bone cement.

One gram of intravenous (IV) tranexamic acid was injected at least 30 minutes before the incision, $1 \mathrm{~g}$ of diluted trenexamic acid to $30 \mathrm{~mL}$ by physiologic serum $(0.9 \% \mathrm{NaCl})$ was given intraarticularly following the closure of the wound, and another $1 \mathrm{~g}$ was infused at the second postoperative hour.

For preemptive analgesia, paracetamol $500 \mathrm{mg}$ tablets were prescribed three times as two tablets per day beginning from three days before the operation. One gram of IV infusion of paracetamol was given just after the operation in postoperative care unit and continued as three times of $1 \mathrm{~g}$ IV infusion. First-line rescue analgesic was intramuscular $75 \mathrm{mg}$ diclofenac sodium and second-line analgesic was IV $100 \mathrm{mg}$ tramadol hydrochloride.

One gram of IV cefazolin sodium was applied 30 minutes before the incision as antibiotic prophylaxis. Low-molecular-weight heparin (enoxaparin sodium) 4,000 IU/0.8 mL/day was used subcutaneously as thromboembolic prophylaxis starting at the postoperative sixth to eighth hours and continued for 20 days.

Patients were mobilized at the fourth hour following surgery and standard physiotherapy program was scheduled during hospitalization (cold-pack once in every 2 hours for 15 minutes, ankle pump exercises, quadriceps isometric exercises, active assisted heel slide exercises in bed, and knee flexion exercises in sitting position/three sets $\times 10$ repeats). Patients were evaluated regularly every two hours during the postoperative period and those fulfilling the discharge criteria were released from the hospital and LOS was recorded for every patient. The standard discharge criteria were as follows: Visual Analog Scale (VAS) score at rest $<3$, VAS score during mobilization $<5$, able to get dressed independently, able to get in and out of bed, able to sit and rise from a chair/toilet seat, independence in personal care, mobilization with walker/crutches, able to walk $>70$ meters without risk of fall with walking aid, no incision problem.

The discharged patients were instructed for a standard home-based exercise program. Patients were also asked to visit the ward at a biweekly interval for the update of the exercise program for the first eight weeks. Fifteen to 40 minutes of walking exercises were also prescribed for five days/week between ninth and twelfth weeks.

\section{Statistical analysis}

Priori power analysis concerning quadriceps muscle strength ${ }^{[10]}$ showed that at an effect size of $\mathrm{d}=0.7,52$ patients are needed (26 patients for each group) to obtain $80 \%$ power (1-beta $=0.80$ ) with $95 \%$ confidence interval (alpha $=0.05$ ).

The data were analyzed using the IBM SPSS Statistics for Windows 24.0 version software (IBM Corp., Armonk, NY, USA). Continuous variables were given as mean \pm standard deviation, median (minimum and maximum) and categorical variable values were presented as absolute numbers and percentages. The conformity of continuous variables with normal distribution was evaluated using the Shapiro-Wilk test. Independent samples t-test for parametric test assumptions and Mann-Whitney $\mathrm{U}$ test for non-parametric test assumptions were used for comparison of the groups. One-way repeatedmeasure analysis of variance was used to compare the normally distributed data from the parameters repeatedly measured in the inner-group analysis, and 


\begin{tabular}{|c|c|c|c|c|c|c|c|c|c|}
\hline \multirow[b]{3}{*}{ Variables } & \multicolumn{8}{|c|}{$\begin{array}{c}\text { TABLE I } \\
\text { Demographic characteristics of patients }\end{array}$} & \multirow[b]{3}{*}{$p$} \\
\hline & \multicolumn{4}{|c|}{ Mini-midvastus } & \multicolumn{4}{|c|}{ Medial parapatellar } & \\
\hline & $\mathrm{n}$ & $\%$ & Mean $\pm S D$ & Min-Max & $\mathrm{n}$ & $\%$ & Mean $\pm S D$ & Min-Max & \\
\hline Age (year) & & & $65.0 \pm 6.4$ & $52-81$ & & & $63.2 \pm 6.3$ & $51-73$ & $0.288(\mathrm{t}=-1.072)$ \\
\hline Height (meter) & & & $1.6 \pm 0.1$ & $1.50-1.78$ & & & $1.6 \pm 0.1$ & $1.47-1.80$ & $0.957(\mathrm{t}=0.055)$ \\
\hline Weight (kilogram) & & & $73.1 \pm 9.8$ & $56-105$ & & & $77.3 \pm 12.0$ & $60-110$ & $0.171(z=-1.369)$ \\
\hline BMI $\left(\mathrm{kg} / \mathrm{m}^{2}\right)$ & & & $28.3 \pm 3.2$ & $22.04-34.13$ & & & $29.8 \pm 3.1$ & $21.77-34.48$ & $0.088(t=1.739)$ \\
\hline \multicolumn{10}{|l|}{ Sex } \\
\hline Female & 26 & 96.3 & & & 24 & 88.9 & & & \\
\hline Male & 1 & 3.7 & & & 3 & 11.1 & & & \\
\hline \multicolumn{10}{|l|}{ Dominant side } \\
\hline Right & 27 & 100 & & & 25 & 92.6 & & & \\
\hline Left & - & - & & & 2 & 7.4 & & & \\
\hline \multicolumn{10}{|l|}{ Operated knee } \\
\hline Right & 12 & 44.4 & & & 12 & 44.4 & & & \\
\hline Left & 15 & 55.6 & & & 15 & 55.6 & & & \\
\hline \multicolumn{10}{|l|}{ Anesthesia } \\
\hline Spinal anesthesia & 12 & 44.4 & & & 12 & 44.4 & & & \\
\hline General anesthesia & 15 & 55.6 & & & 15 & 55.6 & & & \\
\hline
\end{tabular}

Friedman analysis of variance was performed for the remaining data set. Statistical significance was set at $\mathrm{p} \leq 0.05$.

\section{RESULTS}

Demographic characteristics of the patients are given in Table I. Mean LOS was 27.6 \pm 3.1 hours for MMV group and 29.1 \pm 6.7 hours for MPP group. There was no statistical difference between groups in terms of age and LOS. Mean operative time was 78.1 \pm 2.7 minutes for MMV group and 65.9 \pm 2.6 minutes for MPP group. There was a statistically significant difference between groups for the operative time $(p<0.0005)$. There was no statistical difference between groups in terms of hemoglobin and hematocrit values both pre- and postoperatively ( $\mathrm{p}>0.05)$ (Table II).

Preoperative and postoperative fourth and twelfth weeks evaluations of quadriceps muscle strength of operated extremity were significantly

\begin{tabular}{|c|c|c|c|c|c|}
\hline \multicolumn{6}{|c|}{$\begin{array}{l}\text { TABLE II } \\
\text { ostoperative mean values of measured variables }\end{array}$} \\
\hline & \multicolumn{2}{|c|}{ Mini-midvastus } & \multicolumn{2}{|c|}{ Medial parapatellar } & \multirow[b]{2}{*}{$p^{1}$} \\
\hline & Mean $\pm S D$ & Min-Max & Mean \pm SD & Min-Max & \\
\hline \multicolumn{6}{|l|}{ Hemoglobin } \\
\hline Preoperative & $12.9 \pm 1.5$ & $9.5-15.5$ & $13.0 \pm 1.1$ & $10.9-15.6$ & $0.744(\mathrm{t}=0.329)$ \\
\hline Postoperative & $10.3 \pm 1.4$ & $7.2-12.5$ & $10.5 \pm 1.2$ & $8.1-12.1$ & $0.490(t=0.695)$ \\
\hline $\mathrm{P}^{2}$ & \multicolumn{2}{|c|}{$0.000(t: 23.626)$} & \multicolumn{2}{|c|}{$0.000(\mathrm{t}: 14.889)$} & \\
\hline \multicolumn{6}{|l|}{ Hematocrit } \\
\hline Preoperative & $39.4 \pm 3.4$ & $32.6-44.6$ & $39.1 \pm 5.9$ & $31.1-46.70$ & $0.407(\mathrm{t}=0.836)$ \\
\hline Postoperative & $30.7 \pm 5.5$ & $24.60-38.40$ & $32.1 \pm 3.1$ & $26.40-37.40$ & $0.363(t=0.917)$ \\
\hline$P^{2}$ & \multicolumn{2}{|c|}{$0.000(z:-4.542)$} & \multicolumn{2}{|c|}{$0.000(z:-4.543)$} & \\
\hline Length of hospital stay (hour) & $27.6 \pm 3.1$ & $25.06-40.44$ & $29.1 \pm 6.7$ & $25.10-51.32$ & $0.387(z=-0.865)$ \\
\hline Operative time (minute) & $78.1 \pm 2.7$ & $71-82$ & $65.9 \pm 2.6$ & $62-71$ & $0.000(\mathrm{t}=-17.165)$ \\
\hline
\end{tabular}




\begin{tabular}{|c|c|c|c|c|c|}
\hline \multicolumn{6}{|c|}{ TABLE III } \\
\hline & \multicolumn{2}{|c|}{ Mini-midvastus } & \multicolumn{2}{|c|}{ Medial parapatellar } & \multirow[b]{2}{*}{$p^{1}$} \\
\hline & Mean \pm SD & Min-Max & Mean $\pm S D$ & Min-Max & \\
\hline $\begin{array}{l}\text { Operated knee quadriceps muscle } \\
\text { strength (Newton) }\end{array}$ & $107.9 \pm 29.8$ & $52.80-180.66$ & $88.4 \pm 22.7$ & $38.87-131.67$ & $0.011(z=-2.528)$ \\
\hline Preoperative & $127.8 \pm 32.9$ & $67.46-181.33$ & $99.3 \pm 27.2$ & $60.13-186$ & $0.002(z=-3.167)$ \\
\hline $4^{\text {th }}$ week & $131.9 \pm 26.4$ & $100.33-187.33$ & $105.7 \pm 26.9$ & $65.27-172.67$ & $0.002(z=-3.066)$ \\
\hline $\begin{array}{l}12^{\text {th }} \text { week } \\
\mathrm{P}^{2}\end{array}$ & \multicolumn{2}{|c|}{$0.020^{1-3}(F=4.235)$} & \multicolumn{2}{|c|}{$0.024^{1-3}(F=4.025)$} & \\
\hline \multicolumn{6}{|l|}{$\begin{array}{l}\text { Operated knee quadriceps muscle } \\
\text { strength change }\end{array}$} \\
\hline Preoperative and $4^{\text {th }}$ week & $19.9 \pm 44.0$ & $-65.47-93.34$ & $11.0 \pm 35.3$ & $-49.53-99.47$ & $0.415(t=-0.821)$ \\
\hline $4^{\text {th }}$ week and $12^{\text {th }}$ week & $4.1 \pm 47.3$ & $-76.33-78.87$ & $6.4 \pm 30.5$ & $-58.40-82.07$ & $0.836(\mathrm{t}=0.208$ \\
\hline \multicolumn{6}{|l|}{ Operated knee flexion angle } \\
\hline Preoperative & $104.9 \pm 8.7$ & $87-125$ & $104.9 \pm 9.6$ & $85-120$ & $0.976(t=0.030)$ \\
\hline $4^{\text {th }}$ week & $107.2 \pm 10.1$ & $85-125$ & $108.6 \pm 8.0$ & $82-120$ & $0.509(z=-0.660)$ \\
\hline $12^{\text {th }}$ week & $107.3 \pm 8.7$ & $92-125$ & $110.9 \pm 8.5$ & 95-125 & $0.130(\mathrm{t}=1.540)$ \\
\hline $\mathrm{P}^{2}$ & \multicolumn{2}{|c|}{$0.028^{1-3}\left(\chi^{2}=7.173\right)$} & \multicolumn{2}{|c|}{$0.018(F=4.349)$} & \\
\hline \multicolumn{6}{|l|}{ Operated knee extension lag } \\
\hline Preoperative & $-12.3 \pm 4.7$ & $-20--5$ & $-14.6 \pm 7.4$ & $-31--2$ & $0.174(t=-1.380)$ \\
\hline $4^{\text {th }}$ week & $-11.9 \pm 5.7$ & $-25-0$ & $-11.7 \pm 5.9$ & $-20-0$ & $0.870(\mathrm{t}=-0.160)$ \\
\hline $12^{\text {th }}$ week & $-9.8 \pm 4.5$ & $-18--1$ & $-9.5 \pm 6.4$ & $-25-0$ & $0.826(t=0.221)$ \\
\hline $\mathrm{P}^{2}$ & \multicolumn{2}{|c|}{$0.013^{2-3}\left(\chi^{2}=8.747\right)$} & \multicolumn{2}{|c|}{${ }^{*} 0.005^{1-2.1-3}(F=5.793)$} & \\
\hline \multicolumn{6}{|l|}{30 -second chair-stand test } \\
\hline Preoperative & $10.0 \pm 2.6$ & $6-18$ & $9.3 \pm 2.9$ & $2-14$ & $0.358(\mathrm{t}=-0.927)$ \\
\hline $4^{\text {th }}$ week & $10.9 \pm 3.2$ & $6-21$ & $10.0 \pm 2.0$ & $6-16$ & $0.251(z=-1.149)$ \\
\hline $12^{\text {th }}$ week & $12.1 \pm 3.1$ & $7-18$ & $11.1 \pm 2.5$ & $6-17$ & $0.485(z=-0.699)$ \\
\hline $\mathrm{P}^{2}$ & \multicolumn{2}{|c|}{$0.090\left(\chi^{2}=4.822\right)$} & \multicolumn{2}{|c|}{$0.000^{1-3.2-3}\left(\chi^{2}=22.404\right)$} & \\
\hline \multicolumn{6}{|l|}{ Stair-climb test } \\
\hline Preoperative & $25.1 \pm 9.3$ & $11.18-49.50$ & $29.0 \pm 10.1$ & $14.11-55.02$ & $0.092(z=-1.687)$ \\
\hline $4^{\text {th }}$ week & $23.1 \pm 7.0$ & $9.85-40$ & $25.0 \pm 7.8$ & $11.15-46.97$ & $0.467(z=-0.727)$ \\
\hline $12^{\text {th }}$ week & $19.5 \pm 7.3$ & $6.74-32.78$ & $21.7 \pm 8.3$ & $10.12-42.29$ & $0.300(\mathrm{t}=1.046)$ \\
\hline $\mathrm{P}^{2}$ & \multicolumn{2}{|c|}{$0.009^{1-3.2-3}\left(\chi^{2}=9.407\right)$} & \multicolumn{2}{|c|}{$0.000^{1-2.1-3.2 \cdot-3}(\mathrm{~F}=13.145)$} & \\
\hline
\end{tabular}

SD: Standard deviation; Min: Minimum; Max: Maximum; 1 value of between group comparison analyses; t: Independent samples t-test; $z$ : Mann-Whitney U test; 22 value of within group comparison analyses; F, repeated-measure ANOVA; $\chi^{2}$ : Friedman test; 1-2: Preoperative vs. fourth week; 1-3: Preoperative vs. $12^{\text {th }}$ week; 2-3: Fourth week vs. $12^{\text {th }}$ week.

in favor of MMV group; however, there was no significant difference between groups in terms of difference of changes in any time of evaluations $(\mathrm{p}>0.05)$. MMV group had an average of $22.24 \%$ gain and MPP group had $19.6 \%$ gain in quadriceps muscle strength at the final postoperative follow-up in contrast to preoperative values. Operated knee ROM measurements, 30-seceond chair-stand tests, and stair-climb tests did not show any statistical difference between groups ( $p>0.05$ ) (Table III).

The WOMAC and KOOS patient-related activity limitations were given in Table IV and SF-36 quality of life evaluation results were given in Table V. There was no statistically significant difference between groups for pre- and postoperative WOMAC, KOOS, and SF-36 evaluations ( $\mathrm{p}>0.05)$.
Although there was a significant difference between LDFA and LPFA values in preoperative evaluations $(p=0.001$ and $p=0.029$, respectively), postoperative measurements showed no difference between groups ( $p>0.05)$. In addition, HKA angles, femorotibial angle, MPTA, LDTA, and tibial posterior slope angle, and tibiofemoral angle measurements did not show any significant difference between groups in both pre- and postoperative measurements ( $p>0.05)$ (Table VI).

\section{DISCUSSION}

The most important finding of our study is that MMV approach does not have an advantageous effect on the quadriceps muscle strength, pain, function, LOS, and other outcomes in the rapid recovery protocol 


\begin{tabular}{|c|c|c|c|c|c|}
\hline & d postoper & $\begin{array}{l}\text { ABLE IV } \\
\text { e patient rep }\end{array}$ & d outcome & & \\
\hline & Mini- & lastus & Medial & apatellar & \\
\hline & Mean \pm SD & Min-Max & Mean \pm SD & Min-Max & $p^{1}$ \\
\hline WOMAC-pain & & & & & \\
\hline Preoperative & $10.3 \pm 5.4$ & $0-20$ & $11.9 \pm 3.3$ & $7-19$ & $0.176(t=1.373)$ \\
\hline $4^{\text {th }}$ week & $5.0 \pm 3.3$ & $0-11$ & $5.7 \pm 2.8$ & $1-11$ & $0.449(\mathrm{t}=0.762)$ \\
\hline $12^{\text {th }}$ week & $4.8 \pm 4.1$ & $0-16$ & $3.6 \pm 3.1$ & $0-10$ & $0.342(z=-0.951)$ \\
\hline $\mathrm{P}^{2}$ & $0.000^{1-2}$ & $=17.711)$ & $0.000^{1-2,1-3}$ & $F=59.915)$ & \\
\hline WOMAC-stiffne & & & & & \\
\hline Preoperative & $3.3 \pm 2.2$ & $0-8$ & $4.9 \pm 2.1$ & $0-8$ & $0.007(\mathrm{t}=2.833)$ \\
\hline $4^{\text {th }}$ week & $1.6 \pm 1.4$ & $0-4$ & $2.4 \pm 1.7$ & $0-5$ & $0.074(z=-1.784)$ \\
\hline $12^{\text {th }}$ week & $1.8 \pm 1.6$ & $0-6$ & $1.8 \pm 2.1$ & $0-8$ & $0.676(z=-0.418)$ \\
\hline $\mathrm{P}^{2}$ & $0.002^{1-3}$ & $=12.549)$ & $0.000^{1-3}$ & $=16.725)$ & \\
\hline WOMAC-physica & & & & & \\
\hline Preoperative & $32.3 \pm 17.4$ & $0-72$ & $40.7 \pm 8.9$ & $27-57$ & $0.031(t=2.234)$ \\
\hline $4^{\text {th }}$ week & $18.1 \pm 11.7$ & $0-44$ & $17.2 \pm 8.9$ & $2-40$ & $0.755(t=-0.314)$ \\
\hline $12^{\text {th }}$ week & $14.6 \pm 11.1$ & $2-51$ & $12.1 \pm 10.0$ & $0-43$ & $0.349(z=-0.936)$ \\
\hline $\mathrm{P}^{2}$ & $0.000^{1-2}$ & $=20.096)$ & $0.000^{1-2,1-3,}$ & $F=105.669)$ & \\
\hline WOMAC-total & & & & & \\
\hline Preoperative & $45.7 \pm 23.7$ & $2-96$ & $57.5 \pm 12.7$ & $36-80$ & $0.028(t=2.286)$ \\
\hline $4^{\text {th }}$ week & $24.6 \pm 15.4$ & $0-55$ & $25.2 \pm 11.9$ & $5-55$ & $0.875(\mathrm{t}=0.158)$ \\
\hline $12^{\text {th }}$ week & $21.2 \pm 16.2$ & $2-72$ & $17.5 \pm 14.3$ & $0-61$ & $0.377(\mathrm{z}=-0.883)$ \\
\hline $\mathrm{P}^{2}$ & $0.000^{1-2}$ & $=20.824)$ & $0.000(F$ & $.095)^{1-2 ; 1-3}$ & \\
\hline KOOS-pain & & & & & \\
\hline Preoperative & $47.0 \pm 22.2$ & $2.78-88.89$ & $35.0 \pm 16.4$ & $2.78-69.44$ & $0.028(\mathrm{t}=-2.265)$ \\
\hline $4^{\text {th }}$ week & $68.6 \pm 17.8$ & $33.33-100$ & $67.6 \pm 16.4$ & $36.11-100$ & $0.826(\mathrm{t}=-0.221)$ \\
\hline $12^{\text {th }}$ week & $77.9 \pm 18.4$ & $16.67-100$ & $80.9 \pm 17.7$ & $36.11-10$ & $0.504(z=-0.668)$ \\
\hline $\mathrm{P}^{2}$ & $0.000^{1-2,1-3}$ & $\left.\chi^{2}=32.874\right)$ & $0.000^{1-2,1-3,}$ & $\left.x^{2}=40.056\right)$ & \\
\hline KOOS-symptom & & & & & \\
\hline Preoperative & $57.0 \pm 23.9$ & $10.71-92.86$ & $40.2 \pm 19.5$ & $10.71-75$ & $0.007(\mathrm{t}=-2.831)$ \\
\hline $4^{\text {th }}$ week & $74.1 \pm 12.6$ & $46.42-96.43$ & $73.9 \pm 12.9$ & $50-100$ & $0.970(\mathrm{t}=-0.038)$ \\
\hline $12^{\text {th }}$ week & $76.6 \pm 16.4$ & $28.57-100$ & $75.0 \pm 14.7$ & $32.14-100$ & $0.710(\mathrm{t}=-0.374)$ \\
\hline $\mathrm{P}^{2}$ & $0.000^{1-2 ;}$ & $\left.{ }^{2}=35.126\right)$ & $0.000^{1-2 ; 1}$ & $=77.095)$ & \\
\hline KOOS-daily life & & & & & \\
\hline Preoperative & $52.3 \pm 25.8$ & $2.94-100$ & $42.4 \pm 15.0$ & $5.88-76.47$ & $0.0949(\mathrm{t}=-1.716)$ \\
\hline $4^{\text {th }}$ week & $74.7 \pm 17.6$ & $44.11-100$ & $74.2 \pm 15.5$ & $39.71-97.06$ & $0.916(\mathrm{t}=-0.106)$ \\
\hline $12^{\text {th }}$ week & $80.5 \pm 14.0$ & $42.65-100$ & $83.6 \pm 14.3$ & $70.59-100$ & $0.354(z=-0.927)$ \\
\hline $\mathrm{P}^{2}$ & $0.000^{1-2}$ & $=27.844)$ & $0.000^{1-2,1}$ & $=37.589)$ & \\
\hline KOOS-sports an & & & & & \\
\hline Preoperative & $13.3 \pm 24.1$ & $0-100$ & $3.9 \pm 7.4$ & $0-25$ & $0.023(z=-2.278)$ \\
\hline $4^{\text {th }}$ week & $33.7 \pm 24.8$ & $0-85$ & $20.9 \pm 15.6$ & $0-60$ & $0.073(z=-1.794)$ \\
\hline $12^{\text {th }}$ week & $43.9 \pm 24.8$ & $0-85$ & $30.4 \pm 25.2$ & $0-90$ & $0.044(z=-2.015)$ \\
\hline $\mathrm{P}^{2}$ & $0.000^{1-2,1-3}$ & $\left.\chi^{2}=23.426\right)$ & $0.000^{1-2,1}$ & $=29.753)$ & \\
\hline KOOS-quality of & & & & & \\
\hline Preoperative & $28.9 \pm 21.0$ & $0-87.5$ & $22.2 \pm 20.3$ & $0-68.75$ & $0.227(z=-1.208)$ \\
\hline $4^{\text {th }}$ week & $51.4 \pm 20.6$ & $6.25-87.5$ & $53.7 \pm 24.2$ & $12.5-93.75$ & $0.707(\mathrm{t}=0.378)$ \\
\hline $12^{\text {th }}$ week & $56.5 \pm 24.0$ & $0-93.75$ & $63.0 \pm 26.2$ & $18.75-100$ & $0.348(\mathrm{t}=0.946)$ \\
\hline $\mathrm{P}^{2}$ & $0.000^{1-2}$ & $=21.788)$ & $0.000^{1-2,1}$ & $=24.908)$ & \\
\hline
\end{tabular}

SD: Standard deviation; Min: Minimum; Max: Maximum; WOMAC: Western Ontario and McMaster Universities Osteoarthritis Index; KOOS: Knee Injury and Osteoarthritis Outcome Score; $p 1$ value of between group comparison analyses; $t$ : Independent samples t-test; $z$ : Mann-Whitney $U$ test; $p 2$ value of within group comparison analyses; F: Repeated-measure ANOVA; $\chi^{2}$ : Friedman test; ${ }^{1-2}$ : Preoperative vs. fourth week; ${ }^{1-3}$ : Preoperative vs. $12^{\text {th }}$ week; ${ }^{2-3}$ : Fourth week vs. $12^{\text {th }}$ week.

applied TKA patients. However, operative time was found to be shorter in favor of MPP approach in this study.

Quadriceps muscle strength plays an important role in dynamic control of knee joint and distribution of forces on knee joint. Feczko et al. ${ }^{[15]}$ and Lin et al. ${ }^{[16]}$ reported no significant difference between MMV and MPP approaches in terms of postoperative quadriceps muscle strength in traditional protocol applied TKA patients. On the other hand, Yuan et al. ${ }^{[17]}$ included eight prospective randomized controlled trials and eight retrospective studies in their meta-analysis 


\begin{tabular}{|c|c|c|c|c|c|}
\hline \multicolumn{6}{|c|}{$\begin{array}{l}\text { TABLE V } \\
\text { re- and postoperative patient reported outcomes }\end{array}$} \\
\hline & \multicolumn{2}{|c|}{ Mini-midvastus } & \multicolumn{2}{|c|}{ Medial parapatellar } & \multirow[b]{2}{*}{$p^{1}$} \\
\hline & Mean \pm SD & Min-Max & Mean $\pm S D$ & Min-Max & \\
\hline \multicolumn{6}{|c|}{ SF-36 physical functioning } \\
\hline Preoperative & $34.8 \pm 22.1$ & $0-100$ & $22.0 \pm 15.1$ & $0-65$ & $0.016(t=-2.480)$ \\
\hline $4^{\text {th }}$ week & $54.4 \pm 25.2$ & $5-100$ & $58.3 \pm 19.2$ & $25-95$ & $0.527(\mathrm{t}=0.638)$ \\
\hline $12^{\text {th }}$ week & $63.3 \pm 21.6$ & $5-100$ & $67.2 \pm 23.4$ & $10-100$ & $0.529(\mathrm{t}=0.635)$ \\
\hline $\mathrm{P}^{2}$ & \multicolumn{2}{|c|}{$0.000^{1-2,1-3}(F=16.501)$} & \multicolumn{2}{|c|}{$0.000^{1-2,1-3}(F=45.054)$} & \\
\hline \multicolumn{6}{|c|}{ SF-36 role physical } \\
\hline Preoperative & $17.7 \pm 36.5$ & $0-100$ & $5.6 \pm 17.4$ & $0-75$ & $0.142(z=-1.469)$ \\
\hline $4^{\text {th }}$ week & $28.7 \pm 37.8$ & $0-100$ & $31.5 \pm 43.1$ & $0-100$ & $0.931(z=-0.087)$ \\
\hline $12^{\text {th }}$ week & $40.7 \pm 41.7$ & $0-100$ & $56.5 \pm 46.8$ & $0-100$ & $0.303(z=-1.029)$ \\
\hline $\mathrm{P}^{2}$ & \multicolumn{2}{|c|}{$0.093\left(\chi^{2}=4.761\right)$} & \multicolumn{2}{|c|}{$0.0001-3\left(\chi^{2}=19.433\right)$} & \\
\hline \multicolumn{6}{|c|}{ SF-36 role emotional } \\
\hline Preoperative & $43.2 \pm 47.0$ & $0-100$ & $42.0 \pm 49.4$ & $0-100$ & $0.839(z=-0.203)$ \\
\hline $4^{\text {th }}$ week & $42.0 \pm 39.9$ & $0-100$ & $64.2 \pm 47.1$ & $0-100$ & $0.056(z=-1.911)$ \\
\hline $12^{\text {th }}$ week & $50.6 \pm 47.5$ & $0-100$ & $64.2 \pm 46.2$ & $0-100$ & $0.263(z=-1.120)$ \\
\hline $\mathrm{P}^{2}$ & \multicolumn{2}{|c|}{$0.408\left(\chi^{2}=1.794\right)$} & \multicolumn{2}{|c|}{$0.148\left(\chi^{2}=3.825\right)$} & \\
\hline \multicolumn{6}{|l|}{ SF-36 vitality } \\
\hline Preoperative & $47.22 \pm 24.81$ & $15-100$ & $55 \pm 25.9$ & $0-100$ & $0.253(z=-1.144)$ \\
\hline $4^{\text {th }}$ week & $55.55 \pm 22.28$ & $0-100$ & $61.7 \pm 18.5$ & $30-100$ & $0.278(\mathrm{t}=1.096)$ \\
\hline $12^{\text {th }}$ week & $56.85 \pm 22.02$ & $15-100$ & $63.5 \pm 23.9$ & $20-100$ & $0.292(t=1.065)$ \\
\hline $\mathrm{P}^{2}$ & \multicolumn{2}{|c|}{$0.0331-3(F=3.637)$} & \multicolumn{2}{|c|}{$0.690\left(\chi^{2}=0.742\right)$} & \\
\hline \multicolumn{6}{|c|}{ SF-36 mental health } \\
\hline Preoperative & $58.7 \pm 18.6$ & $20-96$ & $64 \pm 26.9$ & $20-100$ & $0.235(z=-1.189)$ \\
\hline $4^{\text {th }}$ week & $68.9 \pm 19.7$ & $28-100$ & $72.6 \pm 18.9$ & $28-100$ & $0.480(t=0.712)$ \\
\hline $12^{\text {th }}$ week & $68.6 \pm 18.4$ & $32-100$ & $73.3 \pm 20.1$ & $24-100$ & $0.370(\mathrm{t}=0.904)$ \\
\hline $\mathrm{P}^{2}$ & \multicolumn{2}{|c|}{$0.004^{1-2,1-3}(F=6.299)$} & \multicolumn{2}{|c|}{$0,193\left(\chi^{2}=3.293\right)$} & \\
\hline \multicolumn{6}{|c|}{ SF-36 social functioning } \\
\hline Preoperative & $61.6 \pm 29.6$ & $0-100$ & $50.1 \pm 30.5$ & $0-100$ & $0.167(\mathrm{t}=-1.403)$ \\
\hline $4^{\text {th }}$ week & $53.2 \pm 34.8$ & $0-100$ & $63.9 \pm 31.1$ & $0-100$ & $0.224(z=-1.216)$ \\
\hline $12^{\text {th }}$ week & $63.4 \pm 31.4$ & $0-100$ & $77.8 \pm 28.2$ & $12.5-100$ & $0.075(\mathrm{z}=-1.782)$ \\
\hline $\mathrm{P}^{2}$ & \multicolumn{2}{|c|}{$0.379(F=0.988)$} & \multicolumn{2}{|c|}{$0.006^{1-3}(F=5.633)$} & \\
\hline \multicolumn{6}{|l|}{ SF-36 bodily pain } \\
\hline Preoperative & $37.0 \pm 22.3$ & $0-100$ & $31.0 \pm 20.7$ & $0-87.5$ & $0.316(z=-1.002)$ \\
\hline $4^{\text {th }}$ week & $49.3 \pm 23.7$ & $10-100$ & $57.3 \pm 27.0$ & $10-100$ & $0.249(\mathrm{t}=-1.165)$ \\
\hline $12^{\text {th }}$ week & $62.1 \pm 25.3$ & $22.5-100$ & $67.8 \pm 24.0$ & $32.5-100$ & $0.448(z=-0.760)$ \\
\hline $\mathrm{P}^{2}$ & \multicolumn{2}{|c|}{$0.000^{1-3}(F=11.466)$} & \multicolumn{2}{|c|}{$0.000^{1-2,1-3}(F=17.845)$} & \\
\hline SF-36 general h & & & & & \\
\hline Preoperative & $57.6 \pm 22.6$ & $5-100$ & $64.3 \pm 26.7$ & $10-100$ & $0.238(z=-1.180)$ \\
\hline $4^{\text {th }}$ week & $70.4 \pm 17.2$ & $35-100$ & $68.7 \pm 22.4$ & $20-100$ & $0.761(\mathrm{t}=-0.306)$ \\
\hline $12^{\text {th }}$ week & $64.8 \pm 20.8$ & $20-100$ & $71.5 \pm 22.9$ & $20-100$ & $0.268(\mathrm{t}=1.120)$ \\
\hline $\mathrm{P}^{2}$ & 0.341 & .154) & 0.290 & $2.477)$ & \\
\hline
\end{tabular}

and reported that quadriceps sparing approach may accelerate early recovery without increasing the risk of malposition of the prosthesis. However, none of the studies included in meta-analysis was perfomed using rapid recovery protocols. There is also other research in the literature reporting shortterm postoperative quadriceps muscle strength in favor of MMV approach without any difference in long-term follow-up. ${ }^{[12,18]}$ Therefore, there is no clearcut consensus in the literature concerning quadriceps muscle strength in MMV and MPP approaches. ${ }^{[11,12,15,16]}$ Even though the fact that quadriceps muscle strength is better with MMV approach only in the early period, this may suggest additional gains in patients undergoing rapid recovery protocol. On the other hand, in our study, we found that the two surgical methods were not superior to each other in terms of quadriceps muscle strength gain. We believe that the rehabilitation program carried out by a single team with the active participation of the patients along with 


\begin{tabular}{|c|c|c|c|c|c|}
\hline \multicolumn{6}{|c|}{$\begin{array}{c}\text { TABLE VI } \\
\text { postoperative measured radiologic variables }\end{array}$} \\
\hline & \multicolumn{2}{|c|}{ Mini-midvastus } & \multicolumn{2}{|c|}{ Medial parapatellar } & \multirow[b]{2}{*}{$p^{1}$} \\
\hline & Mean \pm SD & Min-Max & Mean $\pm S D$ & Min-Max & \\
\hline \multicolumn{6}{|c|}{ Hip-knee-ankle angle (HKA) } \\
\hline Preoperative & $12.3 \pm 3.6$ & $5.9-17.8$ & $13.8 \pm 4.4$ & $6.20-28.10$ & $0.316(z=-1.004)$ \\
\hline Postoperative & $2.7 \pm 1.8$ & $0.5-8.6$ & $3.4 \pm 2.3$ & $0.5-8$ & $0.302(z=-1.032)$ \\
\hline $\mathrm{P}^{2}$ & \multicolumn{2}{|c|}{$0.021(\mathrm{t}=2.467)$} & \multicolumn{2}{|c|}{$0.000(t=12.674)$} & \\
\hline \multicolumn{6}{|l|}{ Femur-tibia angle } \\
\hline Preoperative & $7.0 \pm 4.0$ & $0-13.80$ & $7.8 \pm 4.2$ & $0.5-15.2$ & $0.466(\mathrm{t}=0.735)$ \\
\hline Postoperative & $4.8 \pm 2.8$ & 0- 10.6 & $4.3 \pm 2.3$ & $0.8-8$ & $0.470(\mathrm{t}=-0.727)$ \\
\hline $\mathrm{P}^{2}$ & \multicolumn{2}{|c|}{$0.000(\mathrm{t}=4.624)$} & \multicolumn{2}{|c|}{$0.000(\mathrm{t}=4.624)$} & \\
\hline \multicolumn{6}{|c|}{ Lateral distal femoral angle (LDFA) } \\
\hline Preoperative & $89.1 \pm 3.4$ & $79.90-96.70$ & $91.8 \pm 2.5$ & $86.60-96$ & $0.001(t=3.367)$ \\
\hline Postoperative & $90.0 \pm 2.4$ & $82.90-94$ & $90.6 \pm 1.8$ & $87.90-94.80$ & $0.333(\mathrm{t}=0.976)$ \\
\hline $\mathrm{P}^{2}$ & \multicolumn{2}{|c|}{$0.109(\mathrm{t}=-1.661)$} & \multicolumn{2}{|c|}{$0.005(t=3.069)$} & \\
\hline \multicolumn{6}{|c|}{ Lateral proximal femoral angle (LPFA) } \\
\hline Preoperative & $90.3 \pm 3.3$ & 83-96.5 & $87.2 \pm 7.4$ & $72.90-93.20$ & $0.029(z=-2.181)$ \\
\hline Postoperative & $90.5 \pm 3.4$ & $81.60-99$ & $90.1 \pm 3.5$ & $81.60-96.30$ & $0.692(\mathrm{t}=-0.398)$ \\
\hline $\mathrm{P}^{2}$ & \multicolumn{2}{|c|}{$0.746(\mathrm{t}=-0.348)$} & \multicolumn{2}{|c|}{$0.000(z:-3.544)$} & \\
\hline \multicolumn{6}{|c|}{ Medial proximal tibial angle (MPTA) } \\
\hline Preoperative & $85.7 \pm 2.7$ & $80-95$ & $86.2 \pm 3.5$ & $81-94$ & $0.494(\mathrm{t}=1.221)$ \\
\hline Postoperative & $90.1 \pm 1.8$ & $88-96$ & $89.3 \pm 1.7$ & $84-92$ & $0.092(t=0.096)$ \\
\hline $\mathrm{P}^{2}$ & \multicolumn{2}{|c|}{$0.001(t=5.791)$} & \multicolumn{2}{|c|}{$0.000(t=3.036)$} & \\
\hline \multicolumn{6}{|c|}{ Lateral distal tibial angle (LDTA) } \\
\hline Preoperative & $88.3 \pm 3.5$ & $80.80-94.40$ & $88.4 \pm 4.1$ & $80.30-96.30$ & $0.904(t=0.122)$ \\
\hline Postoperative & $89.2 \pm 2.9$ & $82.90-94.50$ & $88.2 \pm 4.4$ & $73.10-94$ & $0.373(\mathrm{z}=-0.891)$ \\
\hline $\mathrm{P}^{2}$ & \multicolumn{2}{|c|}{$0.197(\mathrm{t}=-1.324)$} & \multicolumn{2}{|c|}{$0.667(\mathrm{t}=0.435)$} & \\
\hline \multicolumn{6}{|c|}{ Tibia posterior inclination angle } \\
\hline Preoperative & $9.1 \pm 3.2$ & 4-16 & $8.7 \pm 4.8$ & $1-19.30$ & $0.698(\mathrm{t}=-0.390)$ \\
\hline Postoperative & $5.4 \pm 1.4$ & $2.8-7.6$ & $5.7 \pm 2.0$ & $1.9-10$ & $0.470(\mathrm{t}=0.729)$ \\
\hline $\mathrm{P}^{2}$ & \multicolumn{2}{|c|}{$0.000(t=6.029)$} & \multicolumn{2}{|c|}{$0.007(t=2.906)$} & \\
\hline \multicolumn{6}{|l|}{ Tibiofemoral angle } \\
\hline Postoperative & $5.8 \pm 2.0$ & $2.1-9.8$ & $6.30 \pm 3.195 .2$ & $1-13$ & $0.466(t=0.735)$ \\
\hline
\end{tabular}

preoperative patient education classes might have played an important role in gaining postoperative quadriceps muscle strength equally in both groups.

Postoperative pain determines function, quality of life, and utmost patient satisfaction in early postoperative period. Huang et al. $^{[18]}$ and Liu et al. ${ }^{[19]}$ compared MPP and MMV approaches with traditional protocols in terms of postoperative pain in their studies and reported no significant difference. On the other hand, some research in the literature reported postoperative pain in favor of MMV approach. ${ }^{[15,20,21]}$ In our study, we did not detect any difference between groups in terms of postoperative pain. Preemptive analgesia protocol, local infiltration analgesia, and effective postoperative pain management protocol might all have an effect on this finding in our study.

Length of hospital stay is one of the major predictors of the success of the rapid recovery protocols. Decreased LOS stay decreases hospital costs as well as postoperative complications. Therefore, minimal invasive approaches are proposed to be used in rapid recovery protocols targeting a possible decrease in hospital stay. However, in our study, there was no significant difference between MMV and MMP approaches in terms of LOS. Success of rapid recovery pain control protocols, preoperative patient education classes, supervised early mobilization and physiotherapy protocols, and application of discharge criteria are thought to be the major factors in our study for shorter LOS compared to similar studies in the literature. ${ }^{[8-10,22,23]}$

Antony-Leo et al. ${ }^{[24]}$ reported better quality of life and joint specific outcome scores in minimal invasive group than MPP group following a structured 12-week rehabilitation care in their double-blind randomized controlled trial. Although authors' 12-week rehabilitation program was similar to our 
12-week rehabilitation program, this randomized controlled trial was not set up with ERAS ${ }^{\circledR}$ protocol, and they compared subvastus approach with MPP in contrast to comparison of MMV approach with MPP in our study. There were no significant differences between MMV and MPP approaches in terms of postoperative knee ROM in our study as it has been reported in most of the other studies with traditional protocols in the literature. ${ }^{[8,11,21,25]}$ Contemporary supervised early physiotherapy protocols have equally resulted in better functional outcomes for both of the approaches. Therefore, in terms of functional outcomes, we ascertained that neither approach has any advantageous effect with rapid recovery protocol set up in this study.

Mean operative time in MMV approach was found to be longer than MMP approach in our study. Similarly, Peng et al. ${ }^{[26]}$ included 19 randomized controlled trials in their meta-analysis and reported an average 18 minutes longer operative time between the minimal invasive subvastus and MPP approaches. Onggo et al. $^{[27]}$ reported a statistically significantly longer operative time and higher mean blood loss in the MMV than MPP approach in their meta-analysis; however, the difference was small and this was not found to be clinically relevant. In their limitations section, authors stated that due to the limited number of randomized controlled trials, only five researches were included in their meta-analyses. In addition, the clinical outcomes in their research were evaluated based on only two studies. Therefore, more studies are needed to compare the clinical outcomes of these two surgical approaches, namely MMV and MPP, for a level I evidence-based conclusion on this topic. MMV surgical approach has a longer learning curve than MMP approach. Shorter surgical incision in MMV approach may limit visibility of the surgical area at the beginning of the surgery particularly in heavier patients, and placement of retractors and cutting guides may be challenging in some cases. Therefore, these factors may lead to longer TKA operative time ${ }^{[8,15,26,27]}$ However, a few researches in the literature have reported similar operative time in both minimal invasive and MPP approaches. ${ }^{[8,21]}$ This may be related to both the longer learning curve of the minimal invasive approaches and the experience of the individual surgeon.

Malalignment of components in TKA may result in functional disability and premature revision of the components. ${ }^{[12]}$ Due to smaller incision and limited visibility of the surgical area, it has been reported in the literature that use of minimal invasive approaches may result in component malalignment. ${ }^{[25]}$ However, most of the authors have reported no significant difference between standard MPP and minimal invasive approaches in terms of component malalignment if experienced surgeons performed the TKA operations. ${ }^{[20,28-30]}$ Yoo et al. ${ }^{[31]}$ also reported similar clinical and radiological outcomes with minimal invasive TKA in obese patients at a minimum of five-year followup. In accordance with the literature, we did not detect any difference between groups in radiologic evaluations of the components. Valgus deformity in the knee may result in further limitation of the visibility of surgical area particularly in lateral site; however, all the knees we operated in our study were in varus and this may be a reason that we could avoid malalignment of the components in our minimal invasive approach. Preoperative careful deformity analysis and performance of all the operations by the same experienced surgeon might all be effective to prevent malalignment of the components in MMV group in our study. In addition, Picard et al. ${ }^{[32]}$ in their study suggested the use of computer assistance such as navigation, patient specific instrumentation or robotic while shifting from standard TKA towards minimal invasive TKA instead of a sudden jump in order not to expose patients to unnecessary risks.

One of the limitations of our study is the lack of evaluation of patients in postoperative period earlier than the fourth week of the operation. Most of the studies in the literature reports better results in early postoperative period in MMV approach, and similar results in late follow-up in terms of pain and function. ${ }^{[12,15,16]}$ Another limitation is that we did not measure intra- and postoperative blood loss in our patients. Instead, we indirectly evaluated this by measuring hemoglobin and hematocrit values.

In conclusion, although minimal invasive approaches are recommended in some of the protocols, we did not detect any advantageous effect of MMV approach over MPP approach for rapid recovery protocol applied TKA patients in terms of pain, function, quality of life evaluations, and LOS. Longer operative time in the MMV approach compared to MPP approach may be considered as a disadvantage.

\section{Acknowledgements}

The authors sincerely thank Prof. Dr. Hakan Erbay for his valuable assistance during the preparation of the rapid recovery protocols, and Hande Şenol for data analysis.

\section{Declaration of conflicting interests}

The authors declared no conflicts of interest with respect to the authorship and/or publication of this article. 


\section{Funding}

Funding was received from Pamukkale University Scientific Project Department for this study (Project No: 2018TIPF017).

\section{REFERENCES}

1. Sever GB, Cankuş C. The long-term results of cemented Oxford unicompartmental knee arthroplasty: A singlecenter experience. Eklem Hastalik Cerrahisi 2019;30:233-40.

2. Agarwala S, Butani M, D'Mello J, Saksena S, Menon A. Decreasing hospital length of stay and enhancing recovery in Total Knee Arthroplasty. J Clin Orthop Trauma 2020;11:122-8.

3. Husted H. Fast-track hip and knee arthroplasty: clinical and organizational aspects. Acta Orthop Suppl 2012;83:1-39.

4. Stowers MD, Manuopangai L, Hill AG, Gray JR, Coleman B, Munro JT. Enhanced Recovery After Surgery in elective hip and knee arthroplasty reduces length of hospital stay. ANZ J Surg 2016;86:475-9.

5. Mora JP, Scuderi GR. Minimally Invasive Total Knee Arthroplasty: Does Surgical Technique Actually Impact the Outcome? Orthop Clin North Am 2020;51:303-15.

6. Aslam MA, Sabir AB, Tiwari V, Abbas S, Tiwari A, Singh P. Approach to total knee replacement: A randomized double blind study between medial parapatellar and midvastus approach in the early postoperative period in asian population. J Knee Surg 2017;30:793-7.

7. Shukla R, Mahajan P, Singh M, Jain RK, Kumar R. Outcome of total knee replacement via two approaches in indian scenario. J Knee Surg 2017;30:174-8.

8. Heekin RD, Fokin AA. Mini-midvastus versus minimedial parapatellar approach for minimally invasive total knee arthroplasty: outcomes pendulum is at equilibrium. J Arthroplasty 2014;29:339-42.

9. Karpman RR, Smith HL. Comparison of the early results of minimally invasive vs standard approaches to total knee arthroplasty: a prospective, randomized study. J Arthroplasty 2009;24:681-8.

10. Chin PL, Foo LS, Yang KY, Yeo SJ, Lo NN. Randomized controlled trial comparing the radiologic outcomes of conventional and minimally invasive techniques for total knee arthroplasty. J Arthroplasty 2007;22:800-6.

11. Dalury DF, Jiranek WA. A comparison of the midvastus and paramedian approaches for total knee arthroplasty. J Arthroplasty 1999;14:33-7.

12. White RE Jr, Allman JK, Trauger JA, Dales BH. Clinical comparison of the midvastus and medial parapatellar surgical approaches. Clin Orthop Relat Res 1999; 367:117-22.

13. Wainwright TW, Gill M, McDonald DA, Middleton RG, Reed M, Sahota O, et al. Consensus statement for perioperative care in total hip replacement and total knee replacement surgery: Enhanced Recovery After Surgery (ERAS $®)$ Society recommendations. Acta Orthop 2020;91:3-19.

14. Scuderi GR, Tria AJ, editors. Surgical techniques in total knee arthroplasty. 1st ed. New York: Springer-Verlag; 2002.

15. Feczko P, Engelmann L, Arts JJ, Campbell D. Computerassisted total knee arthroplasty using mini midvastus or medial parapatellar approach technique: A prospective, randomized, international multicentre trial. BMC Musculoskelet Disord 2016;17:19.
16. Lin WP, Lin J, Horng LC, Chang SM, Jiang CC. Quadricepssparing, minimal-incision total knee arthroplasty: a comparative study. J Arthroplasty 2009;24:1024-32.

17. Yuan FZ, Zhang JY, Jiang D, Yu JK. Quadriceps-sparing versus traditional medial parapatellar approaches for total knee arthroplasty: a meta-analysis. BMC Musculoskelet Disord 2019;20:117.

18. Huang HT, Su JY, Chang JK, Chen CH, Wang GJ. The early clinical outcome of minimally invasive quadriceps-sparing total knee arthroplasty: report of a 2-year follow-up. J Arthroplasty 2007;22:1007-12.

19. Liu H, Mei X, Zhang Z, Sun J. Mini-midvastus versus minimedial parapatellar approach in simultaneous bilateral total knee arthroplasty with 24-month follow-up. Acta Orthop Traumatol Turc 2015;49:586-92.

20. Nestor BJ, Toulson CE, Backus SI, Lyman SL, Foote KL, Windsor RE. Mini-midvastus vs standard medial parapatellar approach: a prospective, randomized, doubleblinded study in patients undergoing bilateral total knee arthroplasty. J Arthroplasty 2010;25(6 Suppl):5-11.

21. Laskin RS, Beksac B, Phongjunakorn A, Pittors K, Davis J, Shim JC, et al. Minimally invasive total knee replacement through a mini-midvastus incision: an outcome study. Clin Orthop Relat Res 2004;428:74-81.

22. Bäthis H, Perlick L, Blum C, Lüring C, Perlick C, Grifka J. Midvastus approach in total knee arthroplasty: a randomized, double-blinded study on early rehabilitation. Knee Surg Sports Traumatol Arthrosc 2005;13:545-50.

23. Verburg H, Mathijssen NM, Niesten DD, Verhaar JA, Pilot P. Comparison of mini-midvastus and conventional total knee arthroplasty with clinical and radiographic evaluation: A prospective randomized clinical trial with 5-year follow-up. J Bone Joint Surg [Am] 2016;98:1014-22.

24. Antony-Leo AP, Arun-Maiya G, Mohan-Kumar M, Vijayaraghavan PV. Structured total knee replacement rehabilitation programme and quality of life following two different surgical approaches - a randomised controlled trial. Malays Orthop J 2019;13:20-7.

25. Karachalios T, Giotikas D, Roidis N, Poultsides L, Bargiotas $\mathrm{K}$, Malizos KN. Total knee replacement performed with either a mini-midvastus or a standard approach: a prospective randomised clinical and radiological trial. J Bone Joint Surg [Br] 2008;90:584-91.

26. Peng $X$, Zhang X, Cheng T, Cheng M, Wang J. Comparison of the quadriceps-sparing and subvastus approaches versus the standard parapatellar approach in total knee arthroplasty: a meta-analysis of randomized controlled trials. BMC Musculoskelet Disord 2015;16:327.

27. Onggo JR, Onggo JD, Hau R. Comparable outcomes in mini-midvastus versus mini-medial parapatellar approach in total knee arthroplasty: a meta-analysis and systematic review. ANZ J Surg 2020;90:840-5.

28. Kazarian GS, Siow MY, Chen AF, Deirmengian CA. Comparison of quadriceps-sparing and medial parapatellar approaches in total knee arthroplasty: a meta-analysis of randomized controlled trials. J Arthroplasty 2018;33:277-83.

29. Haas SB, Cook S, Beksac B. Minimally invasive total knee replacement through a mini midvastus approach: a comparative study. Clin Orthop Relat Res 2004;(428):68-73. 
30. Cheng YC, Wu PK, Chen CF, Chen CM, Tsai SW, Chang MC, et al. Analysis of learning curve of minimally invasive total knee arthroplasty: A single surgeon's experience with 4017 cases over a 9-year period. J Chin Med Assoc 2019;82:576-83.

31. Yoo JH, Oh HC, Park SH, Kim JK, Kim SH. Does obesity affect clinical and radiological outcomes in minimally invasive total knee arthroplasty? Minimum 5-year followup of minimally invasive TKA in obese patients. Clin Orthop Surg 2018;10:315-21.

32. Picard F, Deakin A, Balasubramanian N, Gregori A. Minimally invasive total knee replacement: techniques and results. Eur J Orthop Surg Traumatol 2018;28:781-91. 